\title{
An Effective Virtual Reality based Remedy for Acrophobia
}

\author{
Maria Abdullah \\ Department of Computer Systems Engineering \\ Dawood University of Engineering \& Technology \\ Karachi, Pakistan
}

\author{
Zubair Ahmed Shaikh \\ Department of Computer Sciences \\ Mohammad Ali Jinnah University, \\ Karachi, Pakistan
}

\begin{abstract}
Virtual reality (VR) Exposure Therapy with sophisticated technology has been used in the Psychological treatment. The goal is to design a virtual environment using HCI (HMD) device with an interactive and immersive realistic 3D graphic scenes for exposure therapy of acrophobia that allows patient to sense height and gets used to the fearful feelings.The degree of fear is then used to evaluate the effectiveness of the system before and after therapy with the help of comparison. One may feel a little uneasy and perhaps accelerated heart rate, excessive sweating and shortness of breath, etc. are some of the most common physical symptoms of anxiety upon exposure to height. This extreme or irrational fear of height is called "Acrophobia". The HMI based simulation is used which used the body sensation elucidation as physical symptoms of anxiety upon exposure to height to predict the results. The test reveals that anxiety level decreases from $16 \%$ at first level exposure and $8 \%$ at last level exposure. It is concluded from the results that VR exposure therapy is more effective than real exposure therapy and also the post test for VR exposure therapy were significantly better than post real exposure results. This system provides cost effective solution for rehabilitation environment.
\end{abstract}

Keywords-HMI; virtual reality (VR); HMD; acrophobia; VR exposure therapy; cognitive behavioral therapy; $3 D V R$ environment

\section{INTRODUCTION}

Virtual reality (VR) innovation has been utilized as a part of the mental treatment of acrophobia and has come to command the treatment of various uneasiness issues. It is currently realized that virtual reality introduction treatment VR exposure therapy regimens are profoundly powerful for acrophobia treatment. Acrophobia, or the dread of statures, is described by a silly dread of tallness circumstances bringing about the shirking of such circumstances or the encounter of such circumstances with stamped trouble. This dread for some is persisted with trouble, though for others, the dread is intense to the point that stature circumstances must be maintained a strategic distance from by and large. Acrophobia can be characterized as a nonsensical and exciting apprehension of stature. Numerous specialists have demonstrated victory in diminishing acrophobia utilizing virtual reality. Virtual reality is an incredible innovation, which advances persistently and which must be continually improved. Virtual the truth is a valuable device to treat fears, yet there are additionally different conditions which could be tackled with the assistance of this innovation. We have numerous opportunities, we simply need to continue endeavoring to discover arrangements and accomplish a definitive objective [1].

In VR exposure therapy patients are presented to virtual uneasiness inciting conditions rather than genuine on edge circumstances. VR exposure therapy depends on the presumption that individuals feel 'display' in the virtual condition. Nearness is defined as a mental state or subjective discernment in which, despite the fact that part or the majority of a person's present affair is produced by or potentially filtered through human-influenced innovation. Those taking after focuses highlighted those hugeness of this examination venture and in addition demonstrate the sources from claiming inspiration to those fruition from claiming this study: 1) A lot of people patients would not dealt with since they can't manage the interview fees of a psychotherapist, something like that it might be an expense compelling result. 2) Those tolerant can't make general sessions from claiming treatment due to going by issues or the long run issues, yet toward those recommended framework they could profit at home additionally. 3) The patients who would suffice starting with any kind for fear Hosting an issue should face psychotherapists since they feel bashful along these lines this might make succeed eventually by perusing utilizing this VR exposure therapy treatment. 4) Patients don't require tying to taking every last one of sessions of restoration. At whatever point somebody won't feel easy, he/she might stop that treatment effectively. In contrast to VR exposure therapy. There are sure impediments to the current arrangement of fear treatment. Well-being and control, Wastefulness in treatment and trouble in planning, Hazard to patient's protection, restricted redundancies of dreaded circumstances [2].

The examination purpose incorporates: 1) is to configure actualize a Virtual Reality-based framework which could help will treat or reduce the particular fear; 2) will offer a VRbased three-dimensional stage the place fear patients could straightforwardly convey for the honest to goodness alternately true particular circumstances Also might respond as stated by those provided for circumstance; 3 ) to develop a framework which might catch that tolerant muscle to motions progressively What's more makes as 3D-virtual earth; 4) to test those suggested system looking into Different sorts of fear patients What's more comes about will a chance to be assessed rely on upon distinctive therapeutic parameters in pulse and Circulatory strain. 
This study has four sections. The first section covers the main idea behind the VR and its applications. The second section would cover the immersive virtual reality would entail the hardware and software setups. The third section covers the reality based implementation of VR. The fourth section covers the results presentation and discussions.

\section{LITERATURE REVIEW}

Virtual the truth is a valuable instrument to treat fears and enhancing the nature of individuals' lives. The adaptable Engineering of the VR application will be used to include more modules and features, to treat a bigger scope of fears, for giving treatment under the reconnaissance of the advisor. The specialist will have the probability of controlling powerfully the changing of the earth. A module for examining the advance of treatment could likewise be actualized, in view of the specialist's comments Advances in the field of $3 \mathrm{D}$ illustrations and the expansion in processing power, the constant visual rendering of a virtual world is conceivable in true. Virtual reality gives new systems of perception, making on the qualities of visual portrayals. In a few occurrences, virtual reality can give all the more precisely the enumerating of a few highlights, procedures, et cetera than by different means, which enables it to perform extraordinary close-up examination of a question, perception from an incredible separation, and examination of territories and occasions inaccessible by different means [1], [2].

The developing notoriety of VR has realized a relentless increment in VR content alongside constant advancements in VR gadgets. Specifically, HMD innovation has pulled in noteworthy enthusiasm from industry. Virtual Reality amusements that require VR-particular gadgets like a headmounted show have wind up noticeably prevalent.

It is normal that VR content joined with a HMD will be utilized as a part of a wide assortment of modern parts, for example, video gaming, film what's more, media, instruction, touring, the medicinal services and restorative field, games, and promoting [3].

The cell phone based VR application has gotten a considerable measure of consideration. As a critical piece of the cell phone based VR application, the outline of menu cooperation has noteworthy effect on the client encounter. Just a head-mounted show (HMD) is required for association. With the presumption that eye and head are well facilitated, the head movement and eyes movement is reliable. The eyes look bearing approximates head introduction, and the eyes look beam can be displayed as a virtual intelligent apparatus. Moreover, a touchpad is coordinated into the HMD, the snap and slide operation on the touchpad can be identified. In view of those works, the info and collaboration continuously is accomplished [4].

The two key essential ways to deal with interface with 3D VR condition are Interaction and immersion. The 3D realistic scenes for presentation treatment of acrophobia must have an intuitive practical scene that guarantees profundity of quality and enables patient to detect stature and gets used to the frightful emotions. Encountering a high Sense of Agency, feeling in control when playing out an activity, is basic to guarantee the productivity of cooperation between a client and any innovation intervened application. In Virtual Reality the Sense of Agency is for the most part considered as a key component of the Presence phenomenon. And can be isolated into two components, he feeling and the judgment of organization, and depends on three standards, in particular the standards of need, selectiveness and consistency, influences the level of immersion in VR The enthusiastic reaction a man has to a living space is overwhelmingly influenced by light, shading and surface as space-production components. To confirm whether this marvel could be recreated in a reenacted environment, the creator led an examination in immersive show that used proportionate outline traits of splendor, shading and surface so as to survey to which degree the enthusiastic reaction in a mimicked domain is influenced by similar parameters influencing genuine environments [5], [6].

Uneasiness issue or Phobia is a sort of mental issue which causes apprehension/nervousness took after by nonstop dread of some circumstance or a thing. It is available generally for a traverse of a half year. The influenced individual may experience a considerable measure of inconvenience in specific circumstances or because of particular items [7].

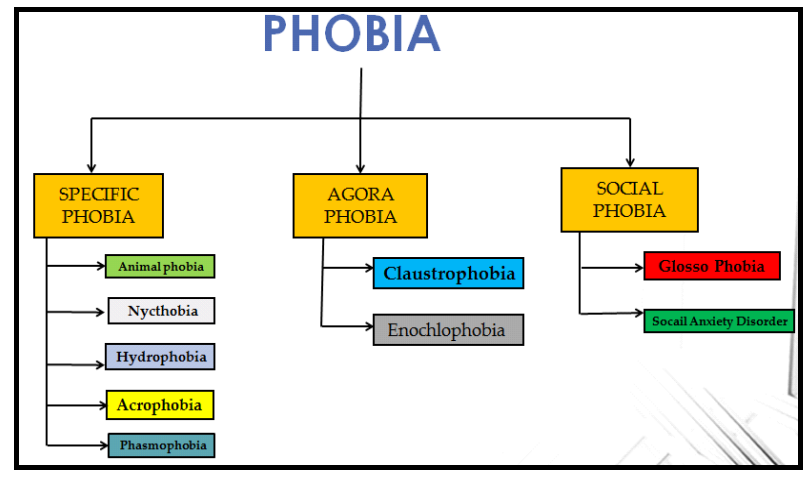

Fig. 1. Categories of Phobia.

Phobia can be separated into different classes, viz. particular or specific, social and agoraphobia as shown in Fig. 1. The most well-known fears are the nervousness because of statures, electrical storms, open talking, lifts and flying. List of different phobias with their associated fear shown in Table I.

TABLE I. List of Phobia with Associated Fear

\begin{tabular}{|l|l|}
\hline SPECIFIC PHOBIA & Fear from specific object and certain situation \\
\hline Animal phobia & Fear of snakes, spider, cockroaches, etc. \\
\hline Nycto phobia & Fear from darkness \\
\hline Hydro phobia & Fear from water \\
\hline Arco phobia & Fear from height \\
\hline Phasmo phobia & Fear of ghosts \\
\hline Haemo phobia & Fear from blood \\
\hline Globo phobia & Fear of balloons \\
\hline AGORA PHOBIA & Cause people to avoid certain places and situation \\
\hline Claustro phobia & Extreme fear of confined places \\
\hline Enochlo phobia & Fear of large crowd and gatherings of people \\
\hline SOCIAL PHOBIA & $\begin{array}{l}\text { Fear of social situations that involves interaction } \\
\text { with people }\end{array}$ \\
\hline Glossoss phobia & Fear of public speaking \\
\hline Social anxiety disorder & $\begin{array}{l}\text { Fear of being judged, negatively evaluated/rejected } \\
\text { in social or performance situation }\end{array}$ \\
\hline
\end{tabular}


Numerous individuals accept there is no actual treatment for fears or that the existing methods of treatment are inefficient. Others are frightened by the thought of confronting the question of dread, giving the fact that a conceivable treatment could involve gradual presentation. There are additionally various treatment techniques which are expensive for most patients or need availability [8], [9].

Therapeutic treatment comprises of tranquilizers and antidepressants that have the objective of reducing nervousness and in this manner helping individuals relax. Unfortunately, they must be utilized with alert, as they can cause dependence [10].

Cognitive Behavioral Therapy (CBT) is the most common method of treatment. CBT has two segments: subjective treatment, which portrays how fear is affected by one's skeptical considerations and behavioral treatment, which contemplates how the sufferer responds when confronting a circumstance causing nervousness [11].

Virtual Reality involves giving our faculties a PC produced virtual condition that we can investigate in some mold. The innovation invigorates two faculties of the client, with a specific end goal to make them experience the VR encounter: sight and hearing [12].

Virtual reality treatment can be compared to the "in vivo" treatment, as it uses similar aspects. It has however the tremendous favorable position of giving a huge combination of conditions and boosts, causing diverse instruments and mental responses. It isn't the main advantage, as virtual reality treatment can diminish the length of the treatment, can give extraordinary openness and adaptability. In expansion, patients can feel more secure and be less wary when they need to communicate with a virtual world, rather than standing up to the protest of dread, all things considered [13]. VR-based mediations have been greatly effective for some nervousness clutters, since this innovation empowers in an extremely exact way the terrifying circumstances and incites regular responses from its clients [14]. The vivo sort of treatment comprises in step by step presenting the sufferer to the reason for fear, without any genuine threat. It can be seen at first as the most exceedingly awful dread of the patient, because it includes the real encounter of the genuine protest causing nervousness. The by the specialist amid the whole move. The power of the presentation to the jolts is normally expanded bit by bit until the point that the sufferer begins feeling better, less terrified within the sight of the protest of dread [15].

Our study is based on VR based innovative technology, the purpose of the software we programmed is to facilitate the medical field in combination with emerging technology that helps suffering people to reduce their fear and improve lives.

\section{IMPLEMENTATION}

The implementation method of our study is based on the true immersive virtual reality. The patient is no more connected with the external world, and fully immersed into the computer-generated sight. The system consists of the following software and hardware setup:

\section{A. Application}

A VR application was constructed by using the Unity $3 \mathrm{~d}$ game engine. Unity Asset Store was used to access more features of Unity $3 \mathrm{~d}$ game engine efficiently. The models for the application were designed by using 3D Max. The VR application consists of two realistic virtual environments and can be interact with in the form of levels. The first level consists of mountainous view with heightened mountain scene as shown in Fig. 2.
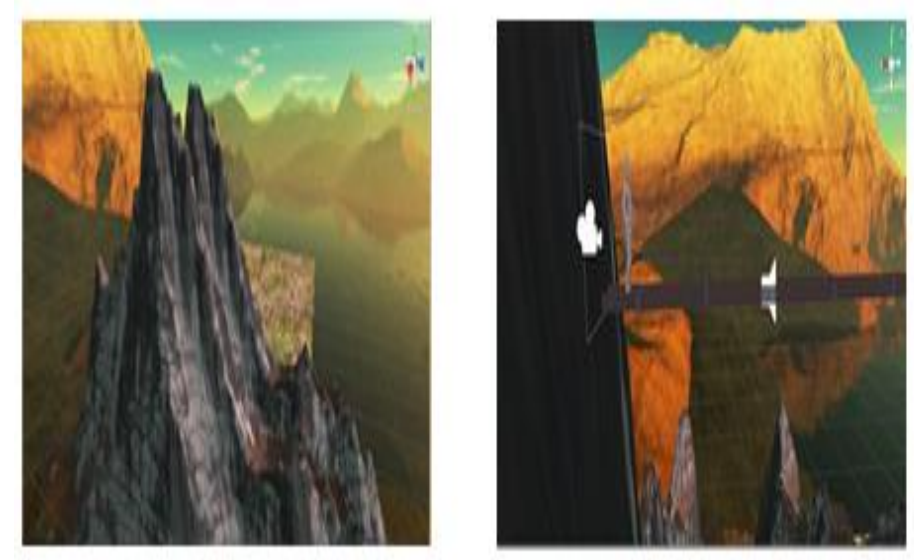

Fig. 2. Mountainous sights.

The second level is comprised of city view with heightened structures as shown in Fig. 3 in both of the environments the models are connected with the heightened channel. The purpose was to build highly immersive sight that triggered patient feared stimulus.
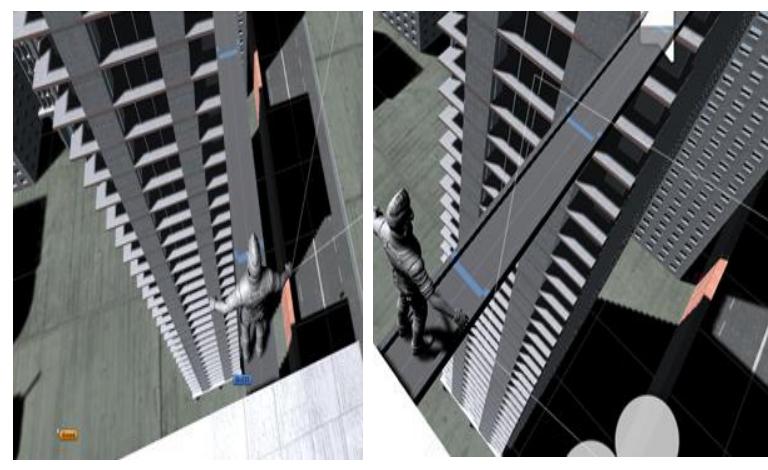

Fig. 3. City sights.

\section{B. Microsoft Kinect Sensor}

Microsoft Kinect with SDK Software development kit is utilized as a part of the venture for detecting the movement of the patient and development following. Its highlights incorporate a RGB camera, a profundity sensor and a multicluster amplifier which conveys a full body $3 \mathrm{~d}$ movement catching, voice acknowledgment and a facial acknowledgment. It is most intense and has propelled capacities. It is additionally good with Unity3D and SDK. The default scope of Kinect is 12 feet with depth sense and color camera. 


\section{VR (HMI) Kit}

Gear VR along with Samsung galaxy S7 edge is used as HMD kit. Virtual environment will be developed using the professional tool Unity 3D. Then the Unity 3D environment will be integrated with HMD to treat the phobias. The Kinect is connected with the PC by using Kinect through SDK and Wireless network is used to for communication between PC and VR kit. The Kinect sense the motion of the patient and the corresponding movement will update in VR environments through wireless networking. The setup flow is shown in Fig. 4.

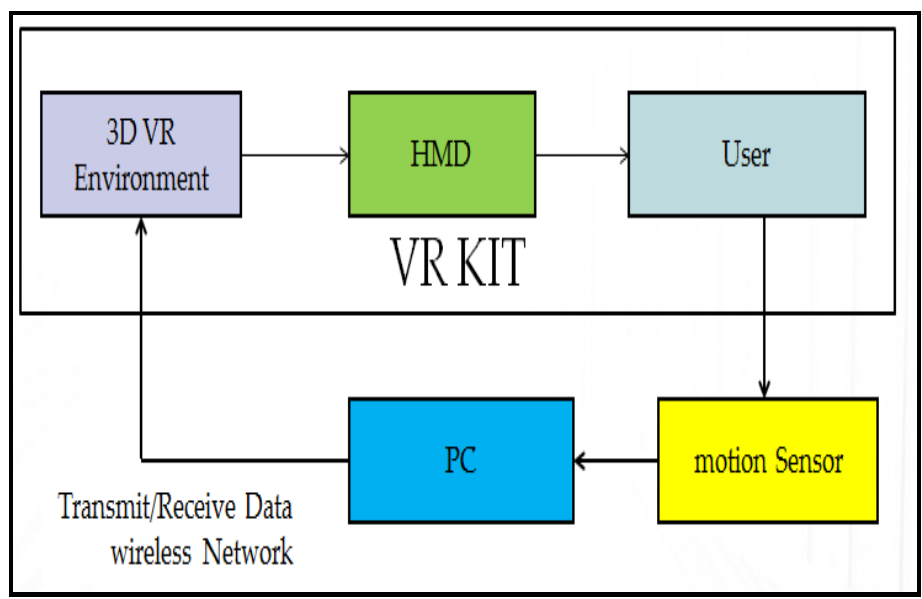

Fig. 4. Setup flow of hardware and software.

\section{METHODOLOGY}

This investigation is to determine the viability of virtual reality presentation treatment VR exposure therapy on acrophobic participant and further to evoked effectiveness by the real exposure treatment through VR exposure therapy. The sample size of is one hundred for this study through Severity Measure for Height Phobia Questionnaire between age group of 20 to 22 years. There were 65 female and 35 male participants responded randomly who were identified as real acrophobic. Results of 20 participants were selected only to make the test simple and effective and also they were agreed to be a part of study if the further testing is required. Afterwards twenty were divided into the group of ten on the basis of having almost same anxiety level. Additionally, Body Sensation Interpretation Questionnaire (BSIQ) is also conducted to find the physical indication of Acrophobic patient on selected participants. In this study, the therapy consisted of eight sessions of each 25 minutes duration was also given to the ten patients (only 10 agreed to participate) for two months with single session per week. The first group was selected for real exposure and the second group was directed to VR exposure therapy. Virtual exposure therapy consisted of two different exposure levels. Four sessions per month for each level with one session per week. Post Exposure surveys were conducted for each group to evaluate the effectiveness of our proposed system.

\section{RESUlts}

From the result of our first survey twenty participants were selected, with the anxiety level shown in Fig. 5. The participants in group were selected in a manner that the overall percentage of anxiety level remain same for comparison purpose.

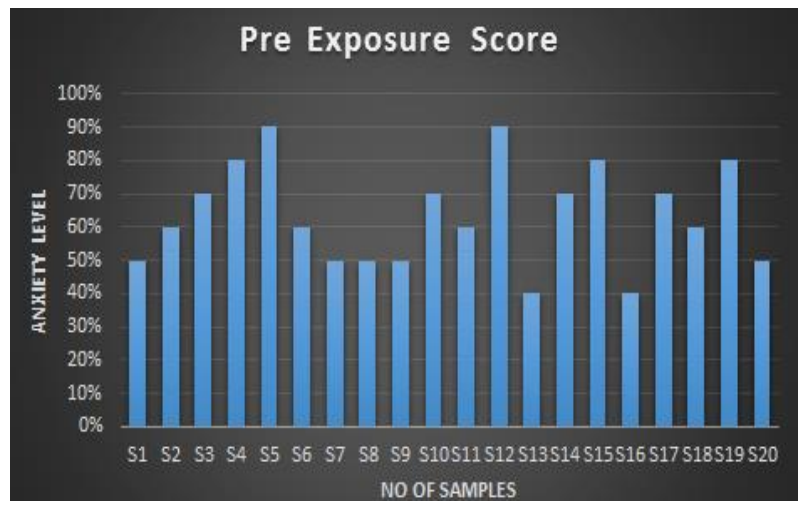

Fig. 5. Pre-exposure anxiety score.

The first group was exposed to the real exposure for two months, with 25 minutes session per week. The post real exposure survey result shows that the anxiety level decreases to a certain level. This can be seen in Fig. 6 .

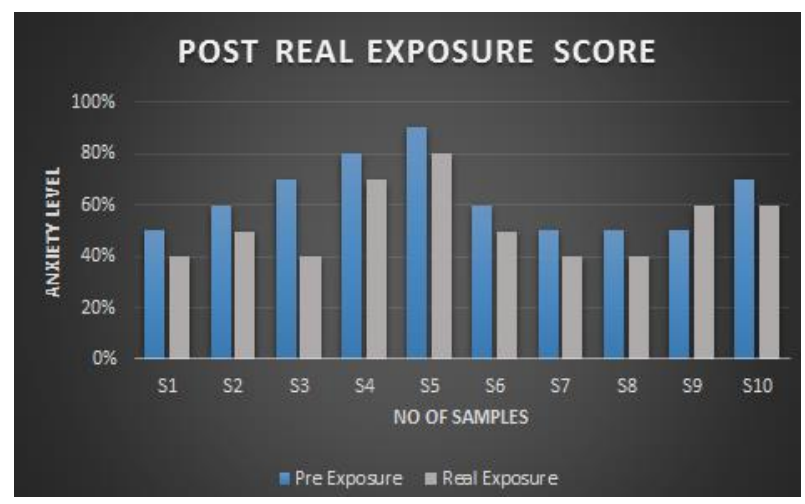

Fig. 6. Post real-exposure anxiety score.

The second group was exposed to the VR exposure for two months, with 25 minutes session per week and one month for each level, the post VR exposure survey result shown in Fig. 7 that the anxiety level decreases to a significant level.

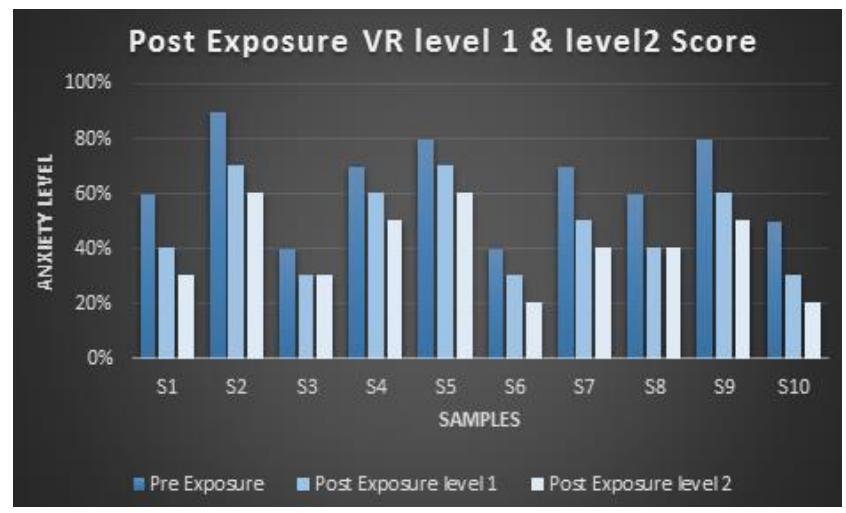

Fig. 7. Post VR-exposure score.

Fig. 8 below shows that the Mean value of Pre vs. Post real Exposure Therapy reduces from $63 \%$ to $53 \%$. 


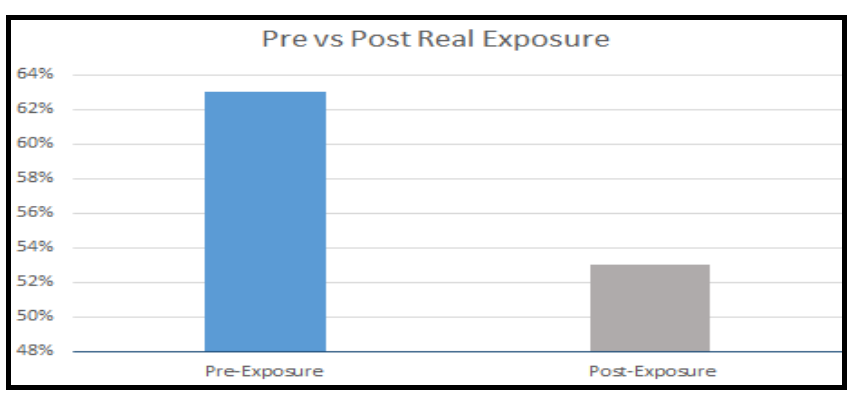

Fig. 8. Mean values of pre and post real-exposure.

Fig. 9 below shows that the Mean value of Pre vs. Post VR Exposure Therapy reduces from $64 \%$ to $48 \%$ during first level exposure of one month. The value further reduces from $48 \%$ to $40 \%$ during second level exposure of one month, with single session per week for both level exposures.

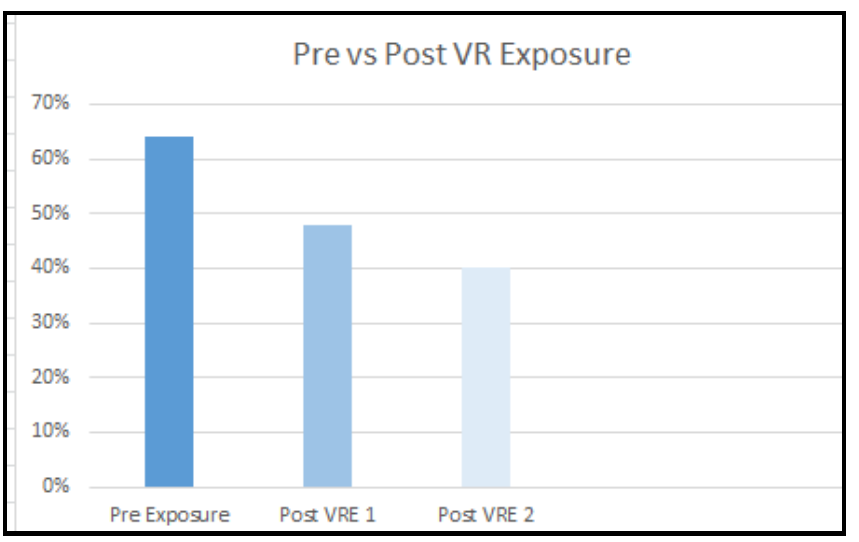

Fig. 9. Mean values of pre \& post VR-exposure.

Fig. 10 shows that the Mean value of BSIQ Score of Pre vs. Post real exposure reduces from 3.0 to 2.6 and the Mean value of Pre vs. Post VR exposure reduces from 2.9 to 2.3.

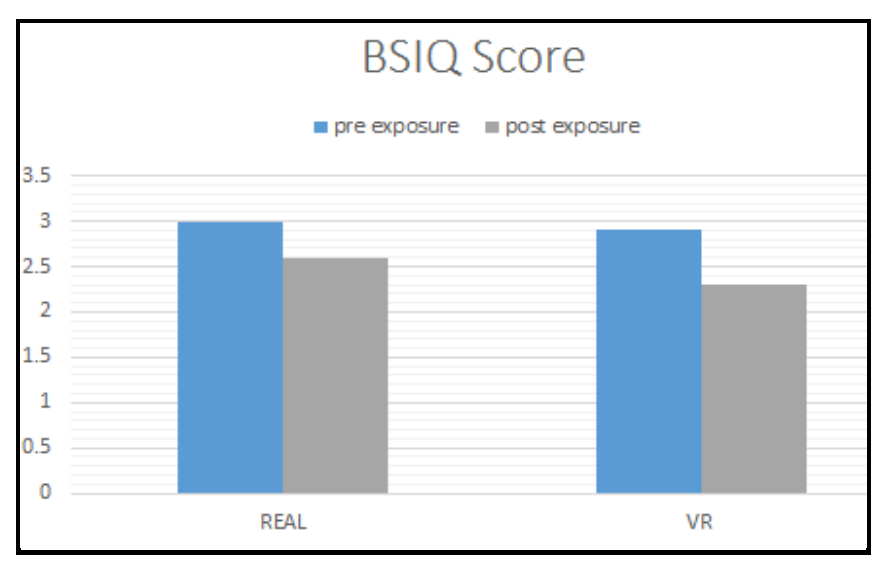

Fig. 10. Comparison of mean values of pre and post exposure.

The results confirmed that the post test for VR exposure therapy were significantly better than post real exposure results. The plots shown in graphs for the post real exposure vs. Post VR exposure also provide the evidence that on the average the post VR exposure results were much superior to post real exposure results.

\section{CONCLUSION}

It has been analyzed during the real exposure there are some risk factors involved while the utilization of virtual reality based application would give more control to the specialist for the treatment prompting a more productive treatment. Protects the secrecy of the patient, the majority of the patients don't need other individuals to think about their apprehensions and since this treatment is directed inside the facility itself there is no hazard of running into companions, family or relatives. Since the sessions are of no longer than thirty minutes hence it allows simplicity of planning. The most vital of these advantages is that it permits the advisor to do boundless reiterations of dreaded circumstances. The anxiety level decreases from $63 \%$ to $53 \%$ after real exposure therapy. .our HMI based simulation involves two levels, the anxiety level decreases from $64 \%$ to $48 \%$ after first level exposure and finally from $48 \%$ to $40 \%$ after last level exposure. It is concluded from the results that VR exposure therapy is more effective than real exposure therapy. The Mean value of BSIQ Score of Pre vs. Post real exposure reduces from 3.0 to 2.6 and the Mean value of Pre vs. Post VR exposure reduces from 2.9 to 2.3. The results confirmed that the post test for VR exposure .therapy were significantly better than post real exposure results

\section{FUTURE WORK}

We can enable more immersive user experiences, along with adding more levels to the current phobia and high-quality graphics. Number of participants can be increased in the future to obtain highly accurate results. Different physiological parameters with the panel of medical professionals may be involved. VR-HMI based application can be used to treatment various other anxiety disorders.

\section{REFERENCES}

[1] "An Innovative Solution Based on Virtual Reality to Treat Phobia", Iulia-Cristina Stănică, Maria-Iuliana Dascălu, Alin Moldoveanu and Florica Moldoveanu. International Journal of Interactive Worlds. http://www.ibimapublishing.com/journals/IJIW/ijiw.html Vol. 2017 (2017), Article ID 155350, 13 pages DOI: 10.5171/2017.155350

[2] "A Cost Effective Approach towards Virtual Reality: Phobia Exposure Therapy", Tejas Parab, Deepankar Pawar, Akshay Chaudhari3 IJARCCE - International Journal of Advanced Research in Computer and Communication Engineering, Vol. 5, Issue 3, March 2016

[3] "A Development of Virtual Reality Game utilizing Kinect, Oculus Rift and Smartphone", DongIk Lee, KiYeol Baek, JiHyun Lee, Hankyu Lim International Journal of Applied Engineering Research ISSN 0973-4562 Volume 11, Number 2 (2016)

[4] "A Novel Menu Interaction Method Using Head-mounted Display for Smartphone-based Virtual Reality", Changchong Sheng, Libing Jiang, Bo Tang, and Xiaoan Tang/ 2017 Progress in Electromagnetics Research Symposium - Spring (PIERS), St Petersburg, Russia

[5] "Towards Novel Approaches to Characterize, Manipulate and Measure the Sense of Agency in Virtual Environments" Camille Jeunet, Louis Albert, Ferran Argelaguet, Anatole L'ecuyer/ IEEE Transactions on Visualization and Computer Graphics 17 January 2018, DOI: 10.1109/TVCG.2018.2794598

[6] "Emotional Qualities of VR Space", Asma Naz, Regis Kopper, Ryan P. McMahan, Mihai Nadin/ 2017 IEEE Virtual Reality (VR), Los Angeles, CA, USA

[7] "Virtual Reality: A Possible Technology to Subdue Disorder and Disability". Aditi Gavhane, Gouthami Kokkula, Shubhangi Shinde, Taufiq Monghal and Jignesh Sisodia/ International Conference on 
Global Trends in Signal Processing, Information Computing and Communication 2016.

[8] "Symbolic online exposure for spider fear: Habituation of fear, disgust and physiological arousal and predictors of symptom improvement", Matthews, A., Naran, N. and Kirkby, K.C.,Division of Psychology, School of Medicine, University of Tasmania, Australia/ Journal of Behavior Therapy and Experimental Psychiatry ,03 Jan 2015.

[9] "Psychological approaches in the treatment of specific phobias: A metaanalysis ", Kate B. Wolitzky-Taylor, Jonathan D. Horowitz, Mark B. Powers, Michael J. Telch,/ 2008 Elsevier Ltd.

[10] "An Abrupt Transformation of Phobic Behavior after a Post-Retrieval Amnesic Agent", Marieke SoeterMerel Kind/journal of psychiatrics Neuroscience and Therapeutics. December (2015).

[11] Counseling Directory, Cognitive behavioral therapy, [Online], www.counselling-directory.org.uk
[12] "Psychological response to an emergency in virtual reality: Effects of victim ethnicity and emergency type on helping behavior and navigation" Gamberini L., Chittaro L., Spagnolli A., Carlesso C. /Computers in Human BehaviorElsevier2015, http://dx.doi.org/10.1016/j.chb.2015.01.040

[13] " Outcomes associated with virtual reality in psychological interventions: where are we now?" Turner WA, Casey LM. / Clinical psychol overview, Elsevier. 2014 Dec; Doi: 10.1016/j.cpr.2014.10.003.

[14] "A computational model for the modulation of the prepulse inhibition of the acoustic startle reflex" David Fernando Ramirez-Moreno and Terrence Joseph Sejnowski/ Biological Cybernetics,Springer, March 2012, doi: $10.1007 / \mathrm{s} 00422-012-0485-7$

[15] "Exposure therapy for posttraumatic stress disorder", Joseph, Jeremy S. Gray, Matt J. http://dx.doi.org/10.1037/h0100457 (2008) 